\title{
Secondary charging effects due to icy dust particle impacts on rocket payloads
}

\author{
M. Kassa ${ }^{1}$, M. Rapp ${ }^{2}$, T. W. Hartquist ${ }^{3}$, and O. Havnes ${ }^{1,4}$ \\ ${ }^{1}$ University of Troms $\emptyset$, Institute of Physics and Technology, 9037 Troms $\varnothing$, Norway \\ ${ }^{2}$ Leibniz Institute of Atmospheric Physics, Schlossstrasse 6, 18225 Kühlungsborn, Germany \\ ${ }^{3}$ School of Physics and Astronomy, University of Leeds, Leeds LS29JT, UK \\ ${ }^{4}$ UNIS, University Studies at Svalbard, Longyearbyen, Norway
}

Correspondence to: O. Havnes (ove.havnes@uit.no)

Received: 9 October 2011 - Revised: 6 January 2012 - Accepted: 20 February 2012 - Published: 1 March 2012

\begin{abstract}
We report measurements of dust currents obtained with a small probe and a larger probe during the flight of the ECOMA-4 rocket through the summer polar mesosphere. The payload included two small dust probes behind a larger dust probe located centrally at the front. For certain phases of the payload rotation, the current registered by one of the small dust probes was up to 2 times the current measured with the larger probe, even though the effective collection area of the larger probe was 4 times that of the small one. We analyze the phase dependence of the currents and their difference with a model based on the assumption that the small probe was hit by charged dust fragments produced in collisions of mesospheric dust with the payload body. Our results confirm earlier findings that secondary charge production in the collision of a noctilucent cloud/Polar Summer Mesospheric Echo (NLC/PMSE) dust particle with the payload body must be several orders of magnitude larger than might be expected from laboratory studies of collisions of pure ice particles with a variety of clean surfaces. An important consequence is that for some payload configurations, one should not assume that the current measured with a detector used to study mesospheric dust is simply proportional to the number density of ambient dust particles. The higher secondary charge production may be due to the NLC/PMSE particles containing multiple meteoric smoke particles.
\end{abstract}

Keywords. Space plasma physics (Instruments and techniques)

\section{Introduction}

Polar Mesospheric Summer Echoes (PMSEs) are radar features formed near the mesopause (e.g. Rapp and Lübken, 2004). Some are associated with noctilucent clouds (NLCs), and it is widely accepted that PMSEs are a consequence of the existence of icy "dust" particles in the vicinity of the polar summer mesopause (e.g. Kirkwood et al., 2010). Havnes et al. (1996) first reported the detection of charged PMSE dust particles with a rocket-borne probe launched in an effort to establish the relationship between the PMSE signals and the charge carried by dust.

Glancing impacts of dust particles, having speeds of about $1 \mathrm{~km} \mathrm{~s}^{-1}$, on surfaces can cause the transfer of charge to the particles and their fragments. Rocket-borne probes passing through NLCs and regions associated with PMSEs experience such particle impacts. Some measurements made in the past with probes used to study the charges that dust particles carry in NLC and PMSE regions have most likely been affected by such secondary charging (Zadorozhny et al., 1993; Havnes et al., 1996, 2009; Vostrikov et al., 1997; Gumbel and Witt, 1998; Smiley et al., 2006; Arnyx et al., 2008).

Laboratory experiments have been conducted to investigate collisions of pure ice particles, having sizes of a few $\mathrm{nm}$, with clean surfaces of a variety of materials, including graphite, steel, duralumin, gold, germanium, and fibreglass laminate. They have shown that the ice particles fragment and partly sublimate, and that a small fraction of the fragments that leave a surface can rub charges off it (Dubov and Vostrikov, 1991; Andersson and Pettersson, 1997, 1998; Tomsic, 2003). The probability for producing secondary 


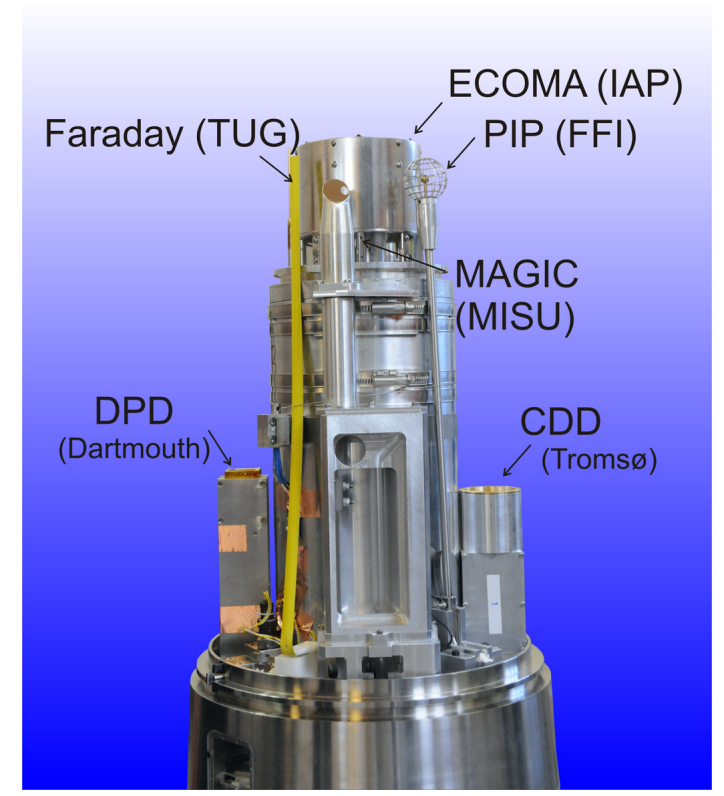

Fig. 1. The front deck of the ECOMA-4 payload, with two small dust particle detectors at its base and the larger IAP dust detector at the top. We consider the currents measured with the IAP and CDD (Troms $\varnothing$ ) UiT dust detectors.

charging in this way is largest for high impact angles, with respect to the normal to the surface. It increases in rough proportion to the surface area of the impacting particle and is about $10^{-3}$ for the largest impacting particles used in the experiments. The charge transfer efficiency also increases with the fragment size, and typically about $10^{-3}$ of the largest fragments leaving a surface carry away an electron (Andersson and Pettersson, 1998; Tomsic, 2003). When a surface is first exposed to an incident beam, negatively chared fragments are predominantly produced but after the surface has been exposed for 5-10 min, the fragments become predominantly positively charged (Tomsic, 2003).

Havnes et al. (1996, 2009) and Havnes and Næsheim (2007) analysed the secondary charging occurring in the DUSTY probe (Havnes et al., 1996) carried on several rocket flights. The coning angle, the angle between the payload axis and the velocity vector, is important for the analysis. Havnes et al. (2009) showed that when the coning angle is small, measurements made at the second grid and bottom plate of the DUSTY probe allow the current associated with the primary charge carried by the impacting particles to be distinguished easily from the current arising due to secondary charging. The DUSTY probe consists of a charge collecting plate behind a set of two biased grids and the current from all are monitored. However, the coning angle of the ECT-07 rocket (Havnes et al., 1996) was not small, which initially led to the dubious conclusion that many incoming primary particles were positively charged. Taking into account the large coning angle, Havnes and Næsheim (2007) found that the observed magnitude and variation, as the payload rotated, of the dust current are consistent with impacting dust particles fragmenting and many of the fragments carrying away an electron each from the grid surface. The removal of electrons by such fragments would produce a false positive contribution to the current onto the grid. Their results indicated that a considerable fraction of the fragments might be of sizes $\sim 1 \mathrm{~nm}$ or even less. The results of Havnes and Næsheim (2007), as well as those of Havnes et al. (1996, 2009), imply that NLC particles with sizes of around $50 \mathrm{~nm}$ may each rub off 50 to 100 electrons. That number of electrons exceeds, by two or three orders of magnitude, the number expected from the scaling of the secondary charging efficiency with particle surface area and the laboratory results described in the preceding paragraph.

The inferred high secondary charging efficiency led Havnes and Næsheim (2007) to propose that mesospheric ice contains meteoric smoke particles. It is not possible to rigorously rule out another cause, e.g. the unpolished nature of the surface in the rocket experiment, of the high efficiency. However, while at impacts of a large pure ice particle, most of the smaller fragments apparently sublimate (Tomsic, 2003), many more fragments of "dirty" ice may survive even if most of the water in which the smoke particles were embedded did sublimate. The survival of a great number of distinct fragments might contribute significantly to "dirty" ice inducing highly efficient secondary charging. Whether or not the high efficiency is due to the "dirty" nature of the impacting particles, the importance of that efficiency for the measurement of the charge carried by NLC and PMSE dust makes its confirmation important.

We report the results of an examination of observations made with dust detectors on the ECOMA-4 rocket. Its launch on 30 June 2008 was one of the activities of the "Existence and charge state of meteoric smoke particles in the middle atmosphere (ECOMA)" project (see Rapp et al., 2011, for more details). Figure 1 shows the locations of different instruments on the front deck of the payload. The large dust probe, which is designated the IAP probe (Rapp and Strelnikova, 2009), was at the very front of the payload. The smaller University of Troms $\varnothing$ (UiT) dust probe, which we designate the UiT probe or the CDD for Charged Dust Detector, was situated further back alongside the payload body. The UiTprobe was smaller than, but otherwise identical to, the original DUSTY probe (Havnes et al., 1996). Both the IAP probe and the UiT probe were Faraday cups. Each had two front grids at potentials of $\pm 6.2 \mathrm{~V}$ to screen the bottom detector plate from external ions and electrons while allowing dust particles to pass.

We examine the consequences of the assumption that the small UiT probe was "sprayed" with charged fragments of the dust particles that impacted the payload body above the opening of this probe. The current carried by the charged fragments would have supplemented the current due to the mesospheric dust particles that impacted directly on the 
probe. We discuss the conditions under which the differences between the currents measured with the UiT probe and the IAP probe could be due to such a spray of charged fragments, and whether the measurements of the currents obtained with the two probes provide support for the large secondary charging efficiency inferred by Havnes et al. (1996, 2009) and Havnes and Næsheim (2007).

\section{Observations}

The collecting area of the IAP dust particle detector was approximately 4 times that of the UiT probe. Therefore, if both detectors had collected only directly incoming charged dust particles, one would expect that the value of the current measured with the IAP probe would be at least 4 times the value obtained with the UiT probe. The deflection of small ambient particles away from the probes by the airflow around the payload (Horányi et al., 1999; Hedin et al., 2007) should have led to a greater loss of current onto the UiT probe than onto the IAP probe. The UiT probe could also have been affected by shadowing if the coning of the payload were non-negligible.

Figure 2a shows the net dust current onto the bottom plate of the IAP probe, while Fig. $2 b$ shows the measured current onto the bottom plate of the UiT probe. Figure $2 \mathrm{c}$ shows the current for the bottom plate of the UiT probe obtained following the application of the correction, described by Havnes et al. (2009), for the secondary current produced by dust impacts on the screening grid just above the bottom plate. This is the total dust current due to directly incoming dust, and the secondary fragments formed by impacts on the payload body ahead of the UiT probe. The IAP probe did not measure the current to its screening grid so a similar correction could not be made. Consequently, Fig. 2a shows the uncorrected current measured with the IAP probe.

From Fig. 2 one sees that as the payload passed through the dust layers the UiT probe current varied greatly due to the rotation, but the IAP probe current was much less affected by rotation. At certain phases of the rotation the magnitude of the IAP probe current was much higher than that of the UiT probe current, but at other phases the opposite was true. This is especially apparent for the altitude region around $88 \mathrm{~km}$ where the UiT probe current varied from close to zero to values comparable to those of the IAP probe current. This cannot be due solely to the shadowing of the UiT probe for two reasons. Firstly, information provided by M. Khaplanov (private communication, 2011) on the interpretation of onboard magnetometer readings implies that the coning angle was only about $8^{\circ}$. Secondly, if shadowing alone had been responsible, the maximum ratio of the UiT probe current to the IAP probe current would be 0.25 .

We suggest that the largest magnitudes of the UiT probe current were caused by the probe being hit, or sprayed, by charged collision fragments, formed by primary dust particles impacting on the payload body in front of the UiT
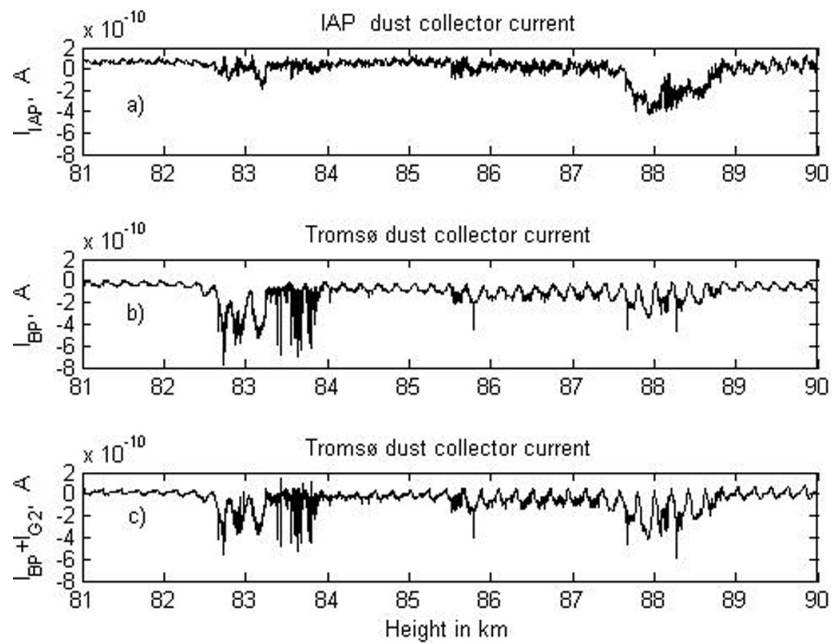

Fig. 2. The currents onto the bottom plates of the IAP and UiT detectors for altitudes from 81 to $90 \mathrm{~km}$. Panels (a) and (b) show measured currents, while panel (c) shows the current to the bottom UiT plate where the contribution from the secondary charging occurring at the screening grid has been removed.

probe. A $180^{\circ}$ change in phase from that corresponding to the largest magnitude of the UiT probe current would have led to that probe experiencing a minimum of collisions with fragments formed by impacts on the payload. However, directly incoming dust particles would still have produced a current, which would have been much smaller in magnitude than that measured with the IAP probe.

\section{Model results}

We model the effects of impacts on the payload body in front of the UiT probe by assuming that the relevant part of the payload body is a $20 \mathrm{~cm}$ long cylindrical tube with a $9 \mathrm{~cm}$ radius, the opening of the UiT probe is at the base of the tube, and the gap between the tube and the UiT probe is $0.5 \mathrm{~cm}$. We will concentrate on two regions at altitudes of around $83 \mathrm{~km}$ and $88 \mathrm{~km}$.

The dust particles at about $83 \mathrm{~km}$ were detectable with radar and a payload photometer and, therefore, may have a mean size of 30 to $50 \mathrm{~nm}$ (Megner et al., 2009; Rapp et al., 2011). We assume a dust number density, $N_{\mathrm{d}}$, of $70 \mathrm{~cm}^{-3}$ for the NLC/PMSE region at $83 \mathrm{~km}$ and that each dust particle there had a charge number, $Z_{\mathrm{d}}$, of -3 . The speed of the rocket there was $V_{\mathrm{R}}=870 \mathrm{~m} \mathrm{~s}^{-1}$ and its coning angle was $\gamma \sim 5^{\circ}$. The upper PMSE region at an altitude of around $88 \mathrm{~km}$ contained smaller dust particles, which were not detectable with the payload photometer. We adopt $N_{\mathrm{d}}=500 \mathrm{~cm}^{-3}$ and $Z_{\mathrm{d}}=-1$ for this PMSE region, where $V_{\mathrm{R}}=800 \mathrm{~m} \mathrm{~s}^{-1}$ and $\gamma \sim 8^{\circ}$. The assumed dust number densities and charge numbers would give a current to the IAP dust probe of $-2 \times 10^{-10} \mathrm{~A}$ in the NLC/PMSE layer and a current 


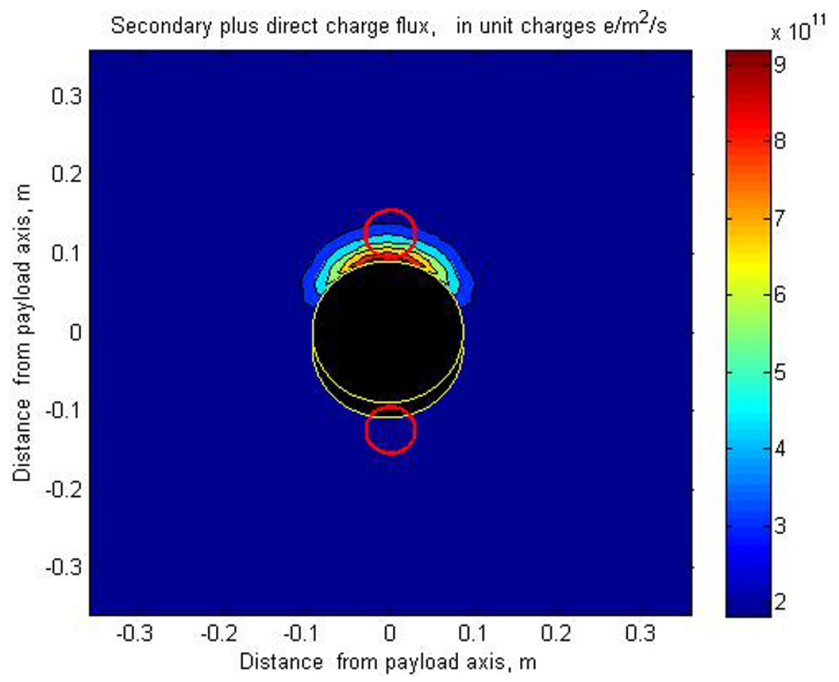

Fig. 3. A typical model distribution of the magnitude of the charge flux, in the plane defined by the entrance to the UiT probe. Most of the flux is due to the secondary charging of ice particle fragments created by impacts on the payload body forward of the UiT probe. The general background flux is the direct flux of ambient charged primary ice particles. The payload cross section is bound by the full yellow circle. The payload shadow in the plane of the opening of the UiT probe is also bound by a closed yellow curve. The cross section of the UiT probe is marked by a red circle and is shown at the positions at which the largest and smallest contributions of secondary charges occur. The magnitude of the flux is given in (unit charges $e$ ) $\mathrm{m}^{-2} \mathrm{~s}^{-1}$. $\eta_{\mathrm{MAX}}=100$ has been assumed. This figure is for the lower NLC/PMSE layer conditions. The results for the upper PMSE layer are very similar, but the shadowed region is slightly larger and the magnitude of the flux is slightly bigger.

of $-5 \times 10^{-10} \mathrm{~A}$ in the higher PMSE layer; the magnitudes of these currents are close to the maximum values measured with the IAP probe as it passed through those layers.

For coning angles of between $\sim 5^{\circ}$ and $\sim 9^{\circ}$, the impact angle is larger than $\sim 81^{\circ}$ and there is no need to consider secondary charge production at lower impact angles. We take $\eta$ to be the number of negative secondary charges produced per primary impact. We assume that it is given by a function that has a maximum, $\eta_{\mathrm{MAX}}$, at an impact angle of $80^{\circ}$ and decreases linearly, with increasing impact angle, to zero for an impact angle of $90^{\circ}$. This behavior is consistent with the variation of secondary production with impact angle described by Tomsic (2003) and Havnes and Næsheim (2007).

We assume that the collision fragments were unaffected by the airflow around the payload body and that each moved in a straight line from the impact point on the tube. Primary dust particles with sizes of less than $\sim 2 \mathrm{~nm}$ would have been swept away from the IAP probe by the shocked airflow in front of the payload (Hedin et al., 2007). However, the only fragments produced on the payload body well behind the shock, but ahead of the UiT probe, that would have been affected significantly by airflow would have been consider- ably smaller. This is due in part to the gas density in most of the relevant region in front of the UiT probe having been lower by a factor of close to 4 than in front of the IAP probe (see Fig. 4.5 in Rapp et al., 2011). Also, the gas speed was significantly lower between the UiT probe and the impact points where secondary charges that hit that probe were created. This resulted in the formation of a weak shock (Rapp et al., 2011), and fragments with sizes well below $1 \mathrm{~nm}$ should have been able to enter the UiT probe. The grid potentials inside the probe would not have significantly affected singly charged collision fragments with sizes of around $1 \mathrm{~nm}$ or more. If many of the secondary charges were carried by fragments that were small enough to be deflected by the airflow around the UiT probe or by its grid potentials, as is suggested by the results of Havnes and Næsheim (2007), our estimate of the secondary charge production would have to be adjusted upwards accordingly. The present measurements give no direct information on the fragment size distribution. At the end of this section we address further the consequences that would follow if the fragments carrying much of the secondary charge were small enough to be affected by airflow and grid potentials.

Collision fragments produced at an impact point would have left it with velocities making angles, to the normal to the surface, that were evenly distributed between $70^{\circ}$ and $90^{\circ}$. These velocities were in the plane defined by the incoming velocity and the normal to the surface at the impact point. Figure 3 shows the distribution of the flux of negative charges in the plane containing the opening of the UiT probe. This plane is normal to the payload, and the flux was due mainly to fragments of the primary ice dust particles that impacted the payload body forward of the UiT probe, but also had a contribution from directly incoming primary dust particles that did not impact the payload body.

Figure 4 shows model results for the dependence, on rotation angle and $\eta_{\max }$, of the total current onto the UiT probe. The total is due to a background of direct impacts and to fragment impacts. The primary particle flux and all other parameters, except the rotation angle, were assumed to be constant. A rotation angle of 0 corresponds to the normal to the opening of the UiT probe being in the plane defined by the payload axis and its velocity and the probe being on the upper, unshadowed side of the payload. At a rotation angle of 0 , the UiT probe is maximally exposed to the collision fragments. The dust density is assumed to be $N_{\mathrm{d}}=70 \mathrm{~cm}^{-3}$, which can be appropriate when a NLC is observable optically and the particle radii are about $50 \mathrm{~nm}$ (von Cossart et al., 1999). Comparisons between the Fig. 4 model results and the observed $83 \mathrm{~km}$ NLC/PMSE UiT probe currents, shown in Fig. 2, indicate that a value of $\eta_{\text {MAX }}$ of about 100 gives a model maximum magnitude of negative current that is comparable to the observed one. This value of $\eta_{\text {MAX }}$ is consistent with the results of an earlier investigation of data from a different rocket payload (Havnes and Næsheim, 2007). 


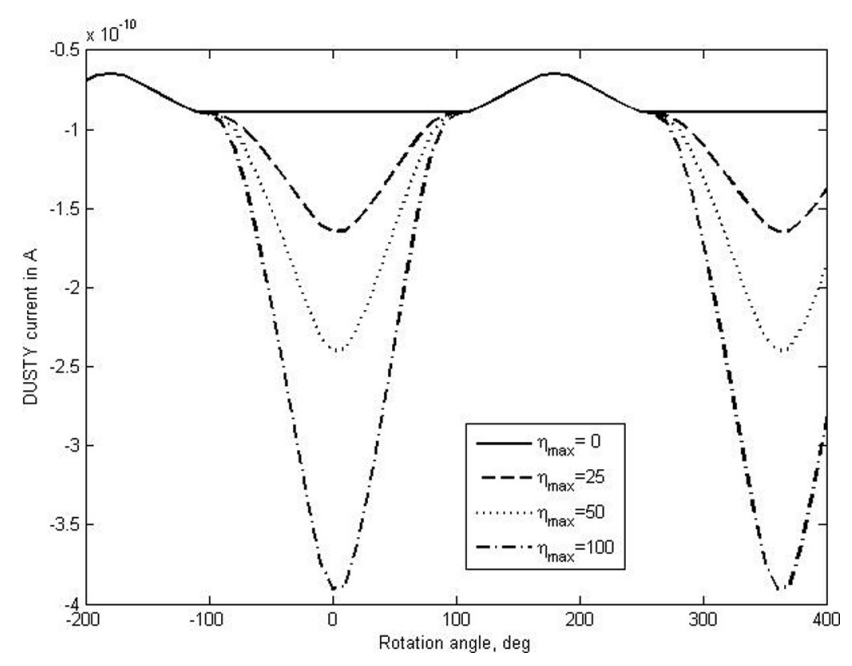

Fig. 4. Model currents onto the UiT probe as functions of rotation angle for the lower, $83 \mathrm{~km}$, NLC/ PMSE layer parameters $\left(N_{\mathrm{d}}=\right.$ $\left.70 \mathrm{~cm}^{-3}, Z_{\mathrm{d}}=-3, \gamma=5.5^{\circ}, V_{\mathrm{R}}=870 \mathrm{~m} \mathrm{~s}^{-1}\right)$ and different values of $\eta_{\text {MAX }}$.

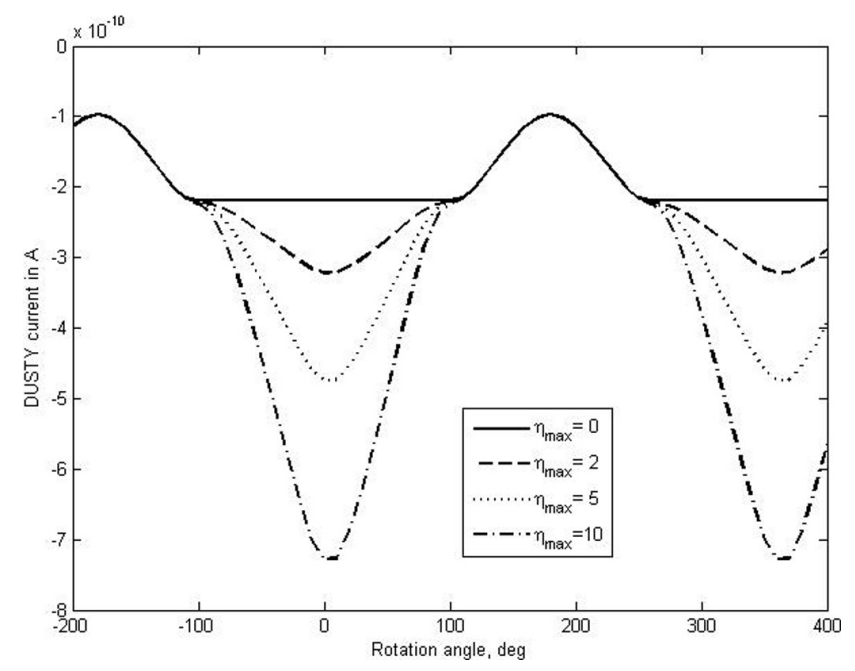

Fig. 5. Model currents onto the UiT probe as functions of rotation angle for the upper, $88 \mathrm{~km}$, PMSE layer parameters $\left(N_{\mathrm{d}}=\right.$ $500 \mathrm{~cm}^{-3}, Z_{\mathrm{d}}=-1, \gamma=8.5^{\circ}, V_{\mathrm{R}}=800 \mathrm{~m} \mathrm{~s}^{-1}$ ) and different values of $\eta_{\text {MAX. }}$.

Figure 5 contains results similar to those in Fig. 4 but which have been obtained for parameters that are more likely to be characteristic of the upper PMSE layer. A value of $\eta_{\max }$ of about 5 leads to reasonable agreement between the model current and the measured UiT probe current, shown in Fig. 2. The value of $\eta_{\max }$ should be smaller for the upper layer than the lower layer, if the secondary charging efficiency scales with particle size, as described in the first section, and the particles in the upper layer are smaller than those in the lower layer, as the optical detection of the lower layer and optical invisibility of the upper layer suggest.

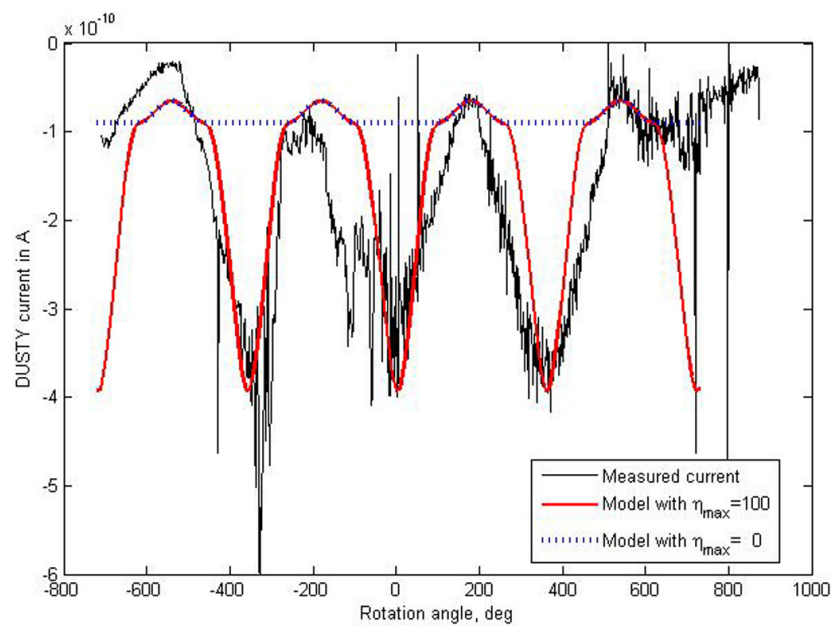

Fig. 6. A comparison between the model profile (red line) for the current onto the UiT probe and the observed profile (black line) for the lower PMSE/NLC layer at $83 \mathrm{~km}$. The profile (blue dotted line) for a model including no secondary production is also shown.

Figure 6 shows a comparison of the model UiT probe current profile, given in Fig. 4, for $\eta_{\max }=100$ with the observed profile (Fig. 2c) for the lower NLC/PMSE layer. The observed variation with rotation is, of course, influenced by changes in dust density with height, which we have neglected. Also, we have not attempted to correct the observed current profile for rotation-related effects other than the shadowing of dust and fragments and secondary charge production, primarily because other such effects are comparatively modest for the lower layer. However, near the upper layer the other rotational effects are larger.

Figure 7 shows the $\sim 86.5 \mathrm{~km}$ to $\sim 89 \mathrm{~km}$ altitude portion of the measured UiT probe current curve, given in Fig. 2c. The rotational variation of the current in the altitude range of $\sim 86.5 \mathrm{~km}$ to $\sim 87.5 \mathrm{~km}$, where there seems to be little dust, is probably due to rotational effects that are not associated with the shadowing of dust and fragments and secondary charge production. We fit the average rotational variation over three rotation periods in that altitude range and assumed that the same variation continued at higher altitudes. The red curve in Fig. 7 gives the fit and its extrapolation to greater altitudes. Figure 8 shows the difference, as a function of rotation angle, between the measured UiT probe current and the fit for the altitude range from $\sim 87.5$ to $\sim 88.3 \mathrm{~km}$. Figure 8 also shows a model UiT current curve for the upper PMSE layer.

Figure 6 shows some differences between the results of our impact-fragmentation model and the observed variations for the lower NLC/PMSE layer. The model current has a somewhat too narrow profile around each of the current minima, which correspond to the maxima in the current magnitude. The current minima occurred when the UiT probe was on the upper side of the payload. In contrast, the model profile is too wide around each of the current maxima, which correspond 


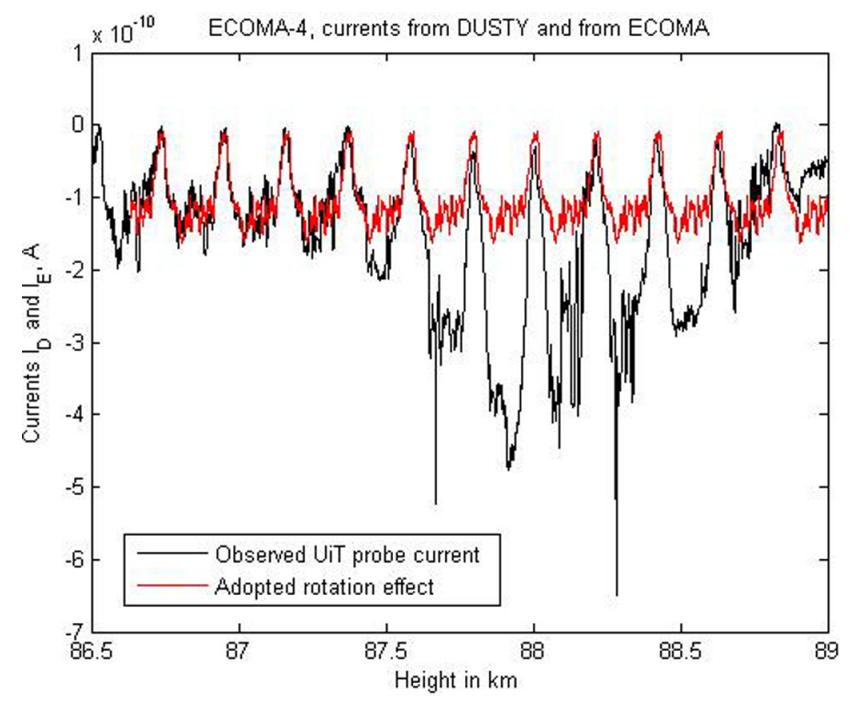

Fig. 7. Fit to the rotational effects unrelated to dust impacts, and the observed UiT dust probe current from Fig. 2c, which is not corrected for these effects.

to the minima in the current magnitude and the times when the probe was partly in the shadow. Although some of these differences may be due to altitude variations of the dust number density and size, the incorporation, in the model, of the influence of the airflow on the fragments would likely bring the model results into better agreement with the observations. Above we presented grounds for assuming that in the airflow would only affect fragments smaller than $\sim 1 \mathrm{~nm}$ in size. Such small fragments may have been created. If they were, the airflow would have deflected them, which would have widened and reduced the amplitude of the profile at each of the minima. Also the deflection of fragments around the payload would have partly filled in the profile around each of the maxima.

Figure 8, for the upper PMSE layer, shows indications that the model profile is too narrow at each of the minima. As noted above, a deflection of collision fragments by the airflow should broaden the profile around a minimum. In contrast at each of the maxima shown in Fig. 8 the observed profile is matched reasonably well by the observed one. The results for the $\eta_{\max }=5$ model shown in Fig. 5 are clearly consistent with the profile near a maximum being affected mostly by the shadowing of primary particles and the profile around a minima being formed mainly by the secondary charged fragments. In the higher altitude case, the model profile around a maximum provides a better match to the data, because in this layer the directly incoming primary dust particles contributed a more significant fraction of the total current to the UiT probe than in the lower layer.

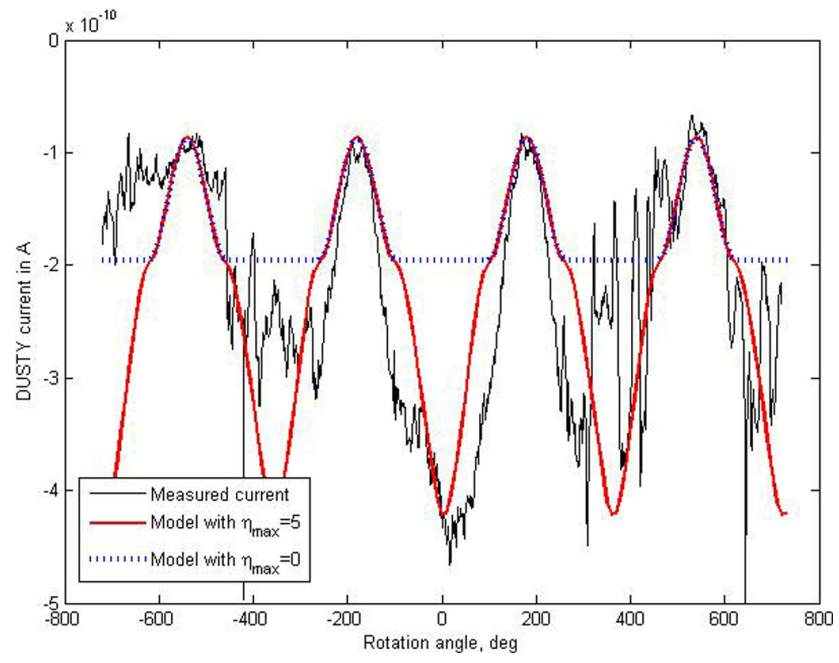

Fig. 8. A comparison between a model profile (red line) and the "corrected" observed profile (black line) for the upper PMSE layer at $88 \mathrm{~km}$. Model results for no secondary production are also shown (blue dotted line).

\section{Conclusions}

For some payload rotation angles, the current measured with the UiT dust probe on the ECOMA-4 rocket was up to twice that measured with the IAP dust probe, despite the IAP probe having had a collecting area that was about 4 times larger than that of the UiT probe. We attribute the substantial differences between the results obtained with the two probes to charge transfer from the payload body to dust fragments created by the impact of ambient mesospheric dust on the payload body in front of the UiT probe. Such fragments did not affect the IAP probe, which was situated in front of the rest of the payload. Model calculations, based on the assumption that our attribution is correct, demonstrate that $\sim 100$ is the required value of the maximum efficiency, $\eta_{\max }$, for the secondary charge production as the payload passed through a NLC/PMSE layer containing optically detected, large dust particles. This value is practically identical to that inferred earlier (Havnes and Næsheim, 2007) in an analysis of data from a different rocket.

The lower value of $\eta_{\max }=5$ for a higher PMSE layer, which was not detected optically, is consistent with that layer containing much smaller dust particles. Experimental results on pure ice (Tomsic, 2003) imply that the secondary charging efficiency varies with impact mass $m_{\mathrm{d}}$ as $m_{\mathrm{d}}^{2 / 3}$, i.e. with the cross section. If we adopt a dust particle size of $50 \mathrm{~nm}$ for the NLC/PMSE layer where, according to our model results, $\eta_{\max }=100$, and assume such a scaling with size, a value of $\eta_{\max }=5$ should imply a dust particle size of $11 \mathrm{~nm}$. This seems reasonable.

An important conclusion is that for some payload configurations, one should not assume that the current measured 
with a detector used to study mesospheric dust is simply proportional to the number density of dust. The large secondary production may also result in dust impacts affecting various probe measurements by influencing the payload potential.

Our results support the suggestion that the mesospheric dust particles can have secondary charge production efficiencies that are at least two orders of magnitude larger than those expected on the basis of the efficiencies, and their scaling with impacting particle size, obtained from laboratory experiments performed with pure ice particles and clean surfaces (e.g. Tomsic, 2003). Havnes and Næsheim (2007) have suggested that the high efficiency of secondary charging is due to each mesospheric NLC/PMSE dust particle containing many meteoric smoke particles embedded in its ice. If such meteoric smoke particles are embedded in the NLC/PMC ice, they might lead to more fragments surviving than would if the NLC/PMSE dust particles were pure ice, because many fragments formed by the impact of pure ice apparently sublimate (Tomsic, 2003). Confirmation of the validity of this suggestion could have consequences for the understanding of the loss mechanisms for metals near the mesopause. Further laboratory experiments could contribute substantially. Some relevant experiments might establish the effects of the cleanliness of the surface on the secondary charging, while others might involve the use of clean surfaces and "dirty" ice particles.

Acknowledgements. MR appreciates support by the German Space Agency under grants 50 OE 0301 and $50 \mathrm{OE} 0801$ (Project ECOMA). The UiT participation on ECOMA with its dust probe was supported by the Norwegian Science Council (NFR).

Topical Editor C. Jacobi thanks M. Friedrich and Z. Sternovsky for their help in evaluating this paper.

\section{References}

Andersson, P. U. and Pettersson, J. B. C.: Ionization of water clusters by collisions with graphite surfaces, Zeitschrift für Physik D, 41, 57-62, doi:10.1007/s004600050289, 1997.

Andersson, P. U. and Pettersson, J. B. C.: Water cluster collisions with graphite surfaces: angular-resolved emission of large cluster ions, J. Phys. Chem. B, 102, 7428-7433, 1998.

Arnyx, K., Sternovsky, Z., Knappmiller, S., Robertson, S., Horanyi, M., and Gumbel, J.: In-situ measurement of smoke particles in the wintertime polar mesosphere between 80 and $85 \mathrm{~km}$ altitude, J. Atmos. Sol.-Terr. Phys., 70, 61-70, 2008.

Dubov, D. Yu. and Vostrikov, A. A.: Collision induced electrification of large water clusters, J. Aerosol Sci., 22, S245-S248, 1991.

Gumbel, J. and Witt, G.: In situ measurements of the vertical structure of a noctilucent cloud, Geophys. Res. Lett., 25, 493-496, 1998.

Havnes, O. and Næsheim, L. I.: On the secondary charging effects and structure of mesospheric dust particles impacting on rocket probes, Ann. Geophys., 25, 623-637, doi:10.5194/angeo25-623-2007, 2007.
Havnes, O., Trøim, J., Blix, T., Mortensen, W., Næsheim, L. I., Thrane, E., and Tønnesen, T.: First detection of charged dust particles in the Earth's mesosphere, J. Geophys. Res., 101, 1083910847, doi:10.1029/96JA00003, 1996.

Havnes, O., Surdal, L. H., and Philbrick, C. R.: Mesospheric dust and its secondary effects as observed by the ESPRIT payload, Ann. Geophys., 27, 1119-1128, doi:10.5194/angeo-271119-2009, 2009.

Hedin, J., Gumbel, J., and Rapp, M.: On the efficiency of rocketborne particle detection in the mesosphere, Atmos. Chem. Phys., 7, 3701-3711, doi:10.5194/acp-7-3701-2007, 2007.

Horányi, M., Gumbel, J., Witt, G., and Robertson, S.: Simulation of rocket-borne particle measurements in the mesosphere, Geophys. Res. Lett., 26, 1537-1540, doi:10.1029/1999GL900298, 1999.

Kirkwood, S., Hervig, M., Belova, E., and Osepian, A.: Quantitative relation between PMSE and ice mass density, Ann. Geophys., 28, 1333-1343, doi:10.5194/angeo-28-1333-2010, 2010.

Megner, L., Khaplanov, M., Baumgarten, G., Gumbel, J., Stegman, J., Strelnikov, B., and Robertson, S.: Large mesospheric ice particles at exceptionally high altitudes, Ann. Geophys., 27, 943951, doi:10.5194/angeo-27-943-2009, 2009.

Rapp, M. and Lübken, F.-J.: Polar mesosphere summer echoes (PMSE): Review of observations and current understanding, Atmos. Chem. Phys., 4, 2601-2633, doi:10.5194/acp-4-2601-2004, 2004.

Rapp, M. and Strelnikova, I.: Measurement of meteor smoke particles during ECOMA-2006 campaign: 1. Particle detection by active photoionization, J. Atmos. Solar-Terr. Physics, 71, 477485, doi:10.1016/j.jastp.2008.06.002, 2009.

Rapp, M., Strelnikova, I., Strelnikov, B.,Friedrich, M., Gumbel, J., Hoppe, U.-P., Blix, T., Havnes, O., Bracikowski, P., Lynch, K. A., and Knappmiller, S.: Microphysical properties of mesospheric aerosols: an overview of results from the ECOMA project, in:Aeronomy of the Earth's Atmosphere and Ionosphere, edited by: Abdu, M. A., Pacheva, D., and Bhattacharyya, A., IAGA Special Sopron Book Series, volume 2, pp. 67-74, Springer Dordrecht Heidelberg, London, New York, 2011.

Smiley, B., Rapp, M., Blix, T. A., Robertson, S., Horanyi, M., Latteck, R., and Fiedler, J.: Charge and size distribution of mesospheric aerosol particles measured inside NLC and PMSE during MIDAS and MaCWAVE 2002, J. Atmos. Solar-Terr. Phys., 68, 114-123, 2006.

Tomsic, A.: Collisions between water clusters and surfaces, Dr. filos. thesis, Gothenburg University, Gothenburg, 2003.

von Cossart, G., Fiedler, J., and von Zahn, U.: Size distributions of NLC particles as determined from 3-color observations of NLC by ground-based lidar, Geophys. Res. Lett., 26, 1513-1516, 1999.

Vostrikov, A. A., Zadorozhny, A. M., Dubov, D. Y., Witt, G., Kazakova, I. V., Bragin, O. A., Kazakov, V. G., Kikhtenko, V. N., and Tyutin, A. A.: Ionization of water clusters by collision with surface, Z. Phys. D, 40, 542-545, 1997.

Zadorozhny, A. M., Tyutin, A. A., Witt, G., Wilhelm, N., Walchli, U., Cho, J. Y. N., and Swartz, W. E.: Electric field measurements in the vicinity of noctilucent clouds and PMSE, Geophys. Res. Lett., 20, 2299-2302, 1993. 